\title{
OCURRENCIA DE Cladosporium EN LA ATMÓSFERA DE LA PLAZA SAN MARTÍN DE LIMA (PERÚ) Y SU RELACIÓN CON ALGUNOS FACTORES METEOROLÓGICOS
}

\section{OCCURRENCE OF Cladosporium IN THE ATMOSPHERE OF THE PLAZA SAN MARTÍN (LIMA, PERU) AND THEIR RELATIONSHIP TO SOME METEOROLOGICAL FACTORS}

\author{
Roberto Ramos ${ }^{1}$ y Víctor Meza ${ }^{2}$
}

\begin{abstract}
Resumen
El objetivo de este estudio fue detectar el hongo alergénico Cladosporium en la atmósfera de la Plaza San Martín de Lima (Perú) y analizar la influencia de las variables meteorológicas sobre su concentración. El muestreo del aire se ha realizado utilizando un equipo de impacto volumétrico tipo Andersen de una sola etapa. Se observó que los conidios de Cladosporium estuvieron presentes en la atmósfera durante todo el período de estudio, sin embargo, las concentraciones más altas se observaron en los meses de marzo y septiembre. Se ha encontrado una correlación estadística positiva entre la concentración de conidios de Cladosporium y la velocidad del viento e índice UV. El efecto de la temperatura del aire presentó una débil correlación positiva sobre la concentración de Cladosporium a diferencia de la humedad relativa que presentó una correlación negativa. La identificación de los factores climáticos que favorecen la liberación de conidios puede mejorar significativamente los métodos actuales de prevención de la alergia, la detección temprana de infecciones en plantas o enfermedades respiratorias en humanos.
\end{abstract}

Palabras clave: bioaerosol, conidios, esporas, alergia, variables climáticas.

\begin{abstract}
The objective of this study was to detect the allergenic fungus Cladosporium in the atmosphere of the Plaza San Martín of Lima (Perú) and to analyze the influence of the meteorological variables on its concentration. Air sampling was performed using a one-stage Andersen type volumetric impact equipment. Cladosporium conidia are present in the atmosphere of Plaza San Martín throughout the study period, however, the highest concentrations were observed in the months of March and September. A positive statistical correlation was found between the concentration of Cladosporium conidia and the wind speed and UV index. The effect of the air temperature showed a weak positive correlation on the concentration of Cladosporium, however, the relative humidity presented a negative correlation. Identification of climatic factors favoring the release of conidia may improve current methods of allergy prevention, early detection of plant infections or respiratory diseases in humans.
\end{abstract}

Key words: bioaerosol, conidia, airborne spore, allergy, meteorological factors.

\section{Introducción.}

Son numerosos los trabajos realizados en el mundo sobre aeromicología (Burch \& Levetin, 2002; Jones \& Harrison, 2004; Stępalska \& Wolek, 2005; Kasprzyk, 2008; Ianovici et al., 2011; Abdel Hameed et al., 2012; Ríos-Yuil et al., 2012; Grinn-Gofroń \& Bosiacka, 2015) que intentan conocer la distribución de los conidios de los hongos de una localidad, su variación en el tiempo y cómo pueden influir determinadas condiciones meteorológicas en su liberación y dispersión.

Estudios realizados en diferentes países indican que los conidios de Cladosporium son los más abundantes en la atmósfera (Rodríguez-Rajo et al., 2005; Kilic et al., 2010; Aira et al., 2012; Grinn-Gofroń \& Strzelczak, 2012) y son los más ampliamente distribuidos en el mundo. También se ha determinado que existe una gran variación estacional en la concentración de conidios de Cladosporium, la más alta concentración ocurre durante el verano (Hasnain, 1993; Burch \& Levetin, 2002; Peternel et al., 2004; Kasprzyk, 2008; Kumari et al., 2011; Grinn-Gofroń \& Strzelczak, 2012). Varios factores meteorológicos como la humedad, temperatura, radiación solar, precipitación, velocidad del viento y presión atmosférica afectan la producción y dispersión conidial en Cladosporium (Sakiyan \& Inceoglu, 2003; Erkara et al., 2008; Kumari et al., 
2011; Zoppas et al., 2011; Grinn-Gofroń \& Bosiacka, 2015).

La inhalación de conidios puede producir síntomas respiratorios alérgicos (Sakiyan \& Inceoglu, 2003; Stępalska \& Wolik, 2005; Erkara et al., 2008; Sen \& Asan, 2009; Viegas et al., 2010; Luo et al., 2016). Los mohos son los alérgenos más comunes y Cladosporium junto con Alternaria son considerados los más predominantes de estos alérgenos (Peternel et al., 2004; Erkara et al., 2008; Luo et al., 2016), por lo que es importante conocer la composición y concentración de los conidios de los hongos en la atmósfera y su relación con las variables meteorológicas. Su conocimiento permitirá, también, la detección temprana de las infecciones en las plantas, favoreciendo así un uso más eficiente y confiable de los plaguicidas (RodríguezRajo et al., 2005).

El objetivo del presente estudio fue detectar al género Cladosporium en la atmósfera de la Plaza San Martín y analizar la influencia de los factores meteorológicos sobre su concentración.

\section{Materiales y métodos.}

Área de estudio.

La ciudad de Lima (Perú) está situada en la costa central del Océano Pacífico $\left(12^{\circ} 2\right.$ ' $45^{\prime}$ ' S, $77^{\circ} 1$ ' 50" O) a $101 \mathrm{~m}$ sobre el nivel del mar. Su clima es de tipo desértico subtropical, tiene una temperatura ambiental que oscila entre $12{ }^{\circ} \mathrm{C}$ en invierno, y $28{ }^{\circ} \mathrm{C}$ en verano, la humedad relativa varía entre 80 y $100 \%$ durante todo el año. La Plaza San Martín se encuentra ubicada en el centro histórico de la ciudad de Lima, centro de desarrollo socioeconómico que concentra más del $50 \%$ de la población nacional. El parque automotor es una de las fuentes principales de emisiones atmosféricas que afectan a las personas que viven en la capital.

Muestreo de la atmósfera.

Las muestras se tomaron en un total de cinco puntos diferentes de la zona de la Plaza San Martín. Los muestreos fueron realizados una vez por mes, de marzo a setiembre de 2014 entre las 10 am y 11 am y se realizaron por el método de impacto utilizando un equipo volumétrico en cascada tipo Andersen de una sola etapa (Andersen, 1958). El flujo de aire aspirado fue de $28.3 \mathrm{l} / \mathrm{min}$ y el muestreo duró 5 minutos por vez y fue ejecutado a $1.5 \mathrm{~m}$ de altura, procedimiento que fue realizado por duplicado de manera secuencial. La concentración de los propágulos en el aire fue expresada en UFC/m³ . Para este cálculo, se utilizó un factor de corrección para el conteo de colonias, basado en la probabilidad de que más de un propágulo viable podría haber pasado a través del mismo agujero e impactado en el medio de cultivo. El cálculo se realizó de acuerdo a la fórmula de Feller (Andersen, 1958):

$$
U F C / m 3=N x \ln [N /(N-P)] / K
$$

Donde $\mathrm{N}$ fue el número total de orificios de la placa, $\mathrm{P}$ es el número de colonias que crecieron en el medio. El resultado se dividió entre un factor de conversión de litros a $\mathrm{m}^{3}(\mathrm{~K}=0.1415)$.

Cultivo e identificación de hongos.

Las placas Petri con agar Sabouraud se incubaron durante 7 días a $25{ }^{\circ} \mathrm{C}$. Las colonias de Cladosporium fueron identificadas hasta género por observación macroscópica y microscópica de las hifas y formas de reproducción asexual. Macroscópicamente las colonias de Cladosporium son de color verde petróleo de aspecto aterciopelado. Microscópicamente, los conidios de Cladosporium son ovoides a cilíndricos y de color verde oliváceo a marrón (Barnett \& Hunter, 1998).

Medida de las variables meteorológicas.

La información meteorológica y atmosférica fue proporcionada por el Servicio Nacional de Meteorología e Hidrología (SENAMHI) a través de su estación meteorológica ubicada en el Campo de Marte situada a $2 \mathrm{Km}$ de la unidad de monitoreo y por el Instituto Metropolitano Protransporte de Lima (PROTRANSPORTE). Se midieron las variables meteorológicas como temperatura, humedad relativa, velocidad del viento e índice UV. Las variables meteorológicas medidas corresponden al momento en que se realizó el experimento.

Análisis estadístico de los datos.

La homogeneidad de varianzas entre la distribución mensual de los conidios de Cladosporium fue analizada por ANOVA de un solo factor. Para determinar la asociación entre los factores meteorológicos y la concentración de conidios de Cladosporium se aplicó el test de correlación de Pearson, con niveles de significancia de 0.05 y 0.01 usando el software estadístico SPSS, versión 20.

\section{Resultados.}

Los propágulos de Cladosporium fueron abundantes y se aislaron durante todo el periodo de estudio en la atmósfera de la Plaza San Martín (Tabla 1). Se observaron dos períodos de mayor concentración, uno durante el mes de marzo y otro durante el mes de setiembre (Figura 1).

Se encontraron diferencias significativas $(p<0.05)$ entre la concentración de esporas y los meses del año. Los meses de marzo y septiembre muestran diferencias

Tabla 1. Concentración de conidios de Cladosporium y variables climáticas en la Plaza San Martín.

\begin{tabular}{lccccccc}
\hline & Marzo & Abril & Mayo & Junio & Julio & Agosto & Setiembre \\
\cline { 2 - 8 } UFC/m & 1382 & 847 & 250 & 594 & 199 & 556 & 1290 \\
Temperatura $\left({ }^{\circ} \mathrm{C}\right)$ & 25.7 & 21.9 & 21.2 & 17.4 & 15.9 & 15.8 & 16.3 \\
Humedad relativa (\%) & 54 & 73 & 74 & 80 & 90 & 89 & 83 \\
Velocidad del viento (m/s) & 1.8 & 1.9 & 1.6 & 1.2 & 0.6 & 1.1 & 1.6 \\
Índice UV & 11.1 & 8.0 & 3.0 & 2.0 & 2.0 & 3.0 & 3.0 \\
\hline
\end{tabular}


Tabla 2. Análisis de correlación de Pearson.

\begin{tabular}{ccccc}
\hline & $\begin{array}{c}\text { Velocidad del viento } \\
(\mathrm{m} / \mathrm{s})\end{array}$ & $\begin{array}{c}\text { Temperatura } \\
\left({ }^{\circ} \mathrm{C}\right)\end{array}$ & $\begin{array}{c}\text { Humedad relativa } \\
(\% \mathrm{HR})\end{array}$ & $\begin{array}{c}\text { Índice } \\
\mathrm{UV}\end{array}$ \\
\cline { 2 - 5 } Concentración de conidios & $\mathrm{r}=0.653$ & $\mathrm{r}=0.425$ & $\mathrm{r}=-0.581$ & $\mathrm{r}=0.657$ \\
$\left(\mathrm{UFC} / \mathrm{m}^{3}\right)$ & $(\mathrm{p}=0.112)$ & $(\mathrm{p}=0.342)$ & $(\mathrm{p}=0.172)$ & $(\mathrm{p}=0.109)$ \\
\hline
\end{tabular}

significativas respecto a los otros meses, así la concentración de propágulos de Cladosporium fue más alta en marzo (1 $382 \mathrm{UFC} / \mathrm{m}^{3}$ ) y setiembre (1 290 $\mathrm{UFC} / \mathrm{m}^{3}$ ) mientras que los valores más bajos se alcanzaron en mayo (250 $\left.\mathrm{UFC}_{\mathrm{m}} \mathrm{m}^{3}\right)$ y julio (199 $\left.\mathrm{UFC} / \mathrm{m}^{3}\right)$. La temperatura del aire fue más alta en marzo $\left(25.7^{\circ} \mathrm{C}\right)$ y abril $\left(21.9^{\circ} \mathrm{C}\right)$, mientras que los valores más bajos fueron en julio $\left(15.9^{\circ} \mathrm{C}\right)$ y agosto $\left(15.8^{\circ} \mathrm{C}\right)$. La velocidad del viento fue más alta en marzo $(1.8 \mathrm{~m} / \mathrm{s})$ y abril $(1.9 \mathrm{~m} / \mathrm{s})$ y los valores más bajos fueron en julio $(0.6 \mathrm{~m} / \mathrm{s})$ y agosto $(1.1 \mathrm{~m} / \mathrm{s})$. También, la humedad relativa fue más alta en julio $(90 \%)$ y agosto $(89 \%)$, los valores más bajos se alcanzaron en marzo (54\%) y abril (73\%). El índice UV fue más alto en marzo (11.1) y abril (8.0) y los valores más bajos fueron en junio (2.0) y julio (2.0). Un resumen de las variables meteorológicas estudiadas y la concentración de conidios de Cladosporium se muestran en la Tabla 1.

Respecto a la influencia de los factores meteorológicos sobre la concentración de propágulos de Cladosporium $\left(\mathrm{UFC} / \mathrm{m}^{3}\right)$, se encontró que la velocidad del viento mostró una correlación positiva

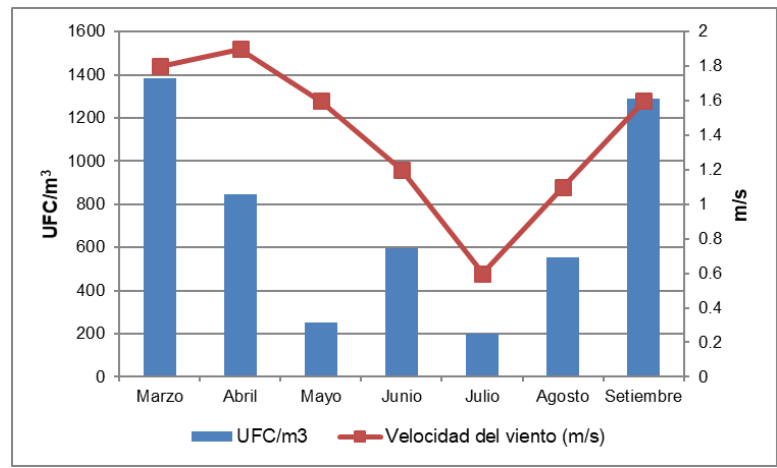

a) Efecto de la velocidad del viento

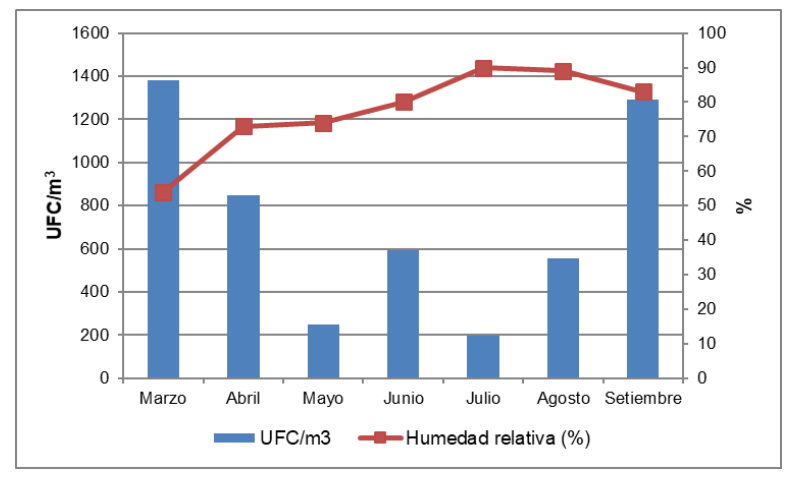

c) Efecto de la humedad relativa $(\mathrm{r}=0.653, \mathrm{p}=0.112)$ (Figura 1a) (Tabla 2), también se ha encontrado que existe una correlación positiva entre la concentración de conidios con el índice UV ( $r=0.657$, $\mathrm{p}=0.109$ ) (Figura 1d). Con relación a las otras variables meteorológicas se ha encontrado que existe una débil correlación positiva entre la concentración de conidios con la temperatura del aire (Figura 1b). Acerca de la humedad relativa (Figura 1c) se encontró que existe una correlación negativa con la concentración de conidios. Las variables climáticas analizadas no presentaron significancia estadística ( $\mathrm{p}>0.05)$ (Tabla 2).

\section{Discusión.}

En nuestro estudio, la velocidad del viento mostró una correlación positiva con el número de conidios de Cladosporium, esto concuerda con Erkara et al. (2008) quienes, usando la correlación de Pearson, observó una correlación positiva. Sin embargo, Lin \& Li (2000), estudiando una zona subtropical urbana, encontraron una fuerte correlación negativa entre la concentración de Cladosporium y la velocidad del viento cuando la velocidad fue por debajo de $5 \mathrm{~m} / \mathrm{s}$. La temperatura

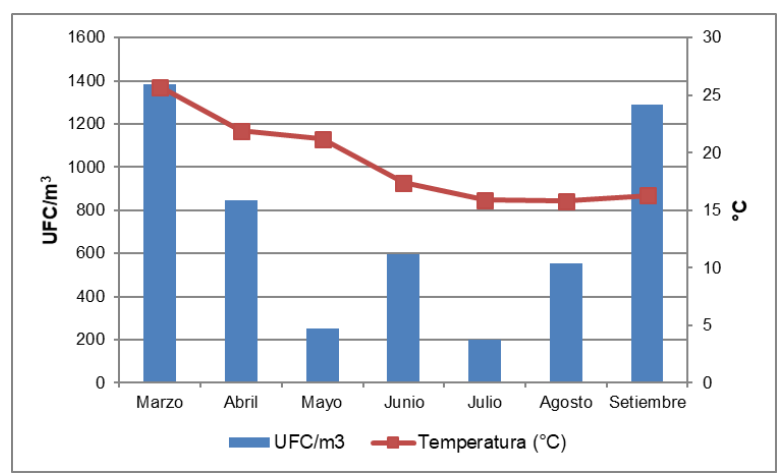

b) Efecto de la temperatura

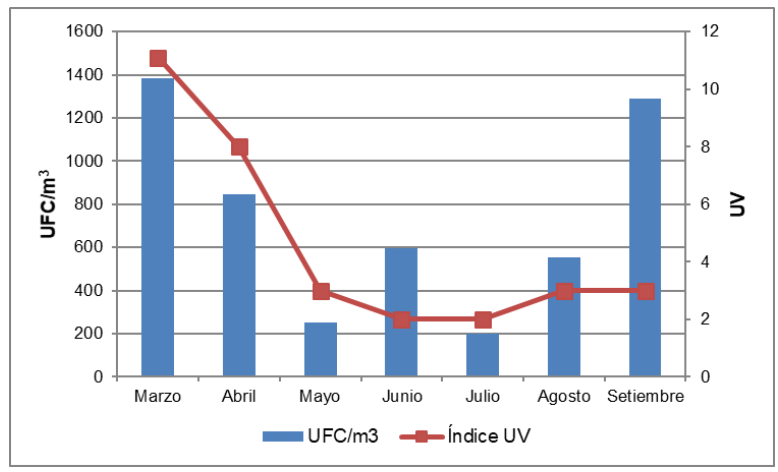

d) Efecto de la luz ultravioleta

Figura 1. Relación entre los parámetros meteorológicos y la concentración de propágulos de Cladosporium. 
presentó una débil correlación positiva sobre la concentración de conidios. Los meses de mayor concentración de Cladosporium en la atmósfera de la Plaza San Martín se producen en los meses de marzo y setiembre, donde la temperatura es más alta y baja respectivamente. (Figuras 1b). La correlación positiva de la velocidad del viento ha sido puesta de manifiesto también por Burch \& Levetin (2002) y por Calderón et al. (1997). Sin embargo, Lyon et al. (1984) encontraron una correlación negativa entre la velocidad del viento y la concentración de conidios. Hjelmroos (1993) mostró una correlación positiva con la temperatura y precipitación. Sabariego et al. (2004) observaron una relación positiva entre la concentración de conidios de Cladosporium y el promedio de las temperaturas máximas y mínimas. Hasnain (1993) encontró una fuerte correlación entre la temperatura máxima y la concentración de Cladosporium. Sánchez \& Almaguer (2014), afirman que, en La Habana, en donde la temperatura varía entre $25{ }^{\circ} \mathrm{C}$ y $30{ }^{\circ} \mathrm{C}$ y la humedad relativa se mantiene por encima del $70 \%$, prevaleció Cladosporium.

Se detectó una correlación negativa entre la humedad relativa y el número de conidios de Cladosporium, la máxima concentración se observó en el mes de marzo cuando la humedad relativa alcanzó el valor más bajo (Figura 1c, Tabla 1), es decir, el número de conidios aumentó cuando los valores de la humedad relativa fueron bajas. Los mismos resultados fueron obtenidos por Aira et al. (2012), en contraste, Katial et al. (1997) halló una correlación positiva entre la humedad relativa y el número de conidios de Cladosporium. Además, se puede observar que existe una relación inversa entre la humedad relativa y la velocidad del viento (Figuras 1c y 1a) en la liberación de los conidios. Dutzmann, (citado por Grinn-Gofron \& Rapiejko, 2009), encontró que la liberación de conidios de Cladosporium por el viento ocurre en conexión con la disminución de la humedad relativa durante las horas de la mañana. Cuando las estructuras que llevan las esporas están suficientemente secas, los conidios maduros serán inmediatamente liberadas por el viento.

El otro parámetro meteorológico, el índice UV, tuvo una correlación positiva alta respecto a las otras variables climáticas. La mayor concentración de conidios coincide con el mayor valor del índice UV (Figuras 1d). Esto indica que la radiación UV estimula la esporulación de Cladosporium, se ha reportado que la luz UV de longitud de onda larga estimula la esporulación de varios hongos (Leach, 1971; Tuite, 1969). La exposición a la luz UV induce la esporulación en hongos (Ramsey \& Bailey, 1930; McCallan \& Chan, 1944), pero recientes estudios han demostrado también que longitudes de onda entre 300 a $380 \mathrm{~nm}$, cercana a la región UV induce más esporulación que aquellas en el rango UV (200-300 $\mathrm{nm})$. Esta última región podría ser letal o mutagénica
(Leach, 1971). Rodríguez-Romero et al. (2010) observaron que la luz ultravioleta $(350-370 \mathrm{~nm})$ y la luz azul (450 nm) actúan como un represor de la reproducción sexual favoreciendo su esporulación.

Varios factores meteorológicos influyen sobre la concentración de los propágulos de Cladosporium en la atmósfera de la Plaza San Martín, tales como la velocidad del viento, humedad e índice UV. Almaguer et al. (2014) afirman que el tamaño de los conidios de este género hace que sean fácilmente aerotransportadas a través de grandes distancias, además asevera que su amplia distribución y liberación de conidios en un amplio rango de temperatura lo convierte en el hongo más abundante en la atmósfera de interiores y exteriores.

\section{Conclusiones.}

La concentración de conidios de Cladosporium en la atmósfera de la Plaza San Martín presenta una distribución con una periodicidad mensual que está determinada por los factores meteorológicos. Existe una débil correlación positiva entre la temperatura del aire y la concentración de conidios, la velocidad del viento y el índice UV presentaron una alta correlación positiva respecto a la concentración de conidios de Cladosporium. Estos resultados sugieren que la presencia y el incremento en la concentración de propágulos de Cladosporium está influenciado por la velocidad del viento y el índice UV.

\section{Agradecimientos.}

A la Municipalidad Metropolitana de Lima e Instituto Metropolitano Protransporte de Lima (PROTRANSPORTE); Servicio Nacional de Meteorología e Hidrología (SENAMHI), y un agradecimiento especial al Laboratorio de Ecología de Artrópodos (LEA) de la UNALM.

\section{Literatura citada.}

Abdel Hameed A. A., Khoder M. I., Ibrahim Y. H., Saeed Y., Osman M. E. \& Ghanem S. 2012. Study on some factors affecting survivability of airborne fungi. Science of the Total Environment, 414, 696-700.

Aira M., Rodríguez-Rajo F., Fernández-González J., Seijo M., C., Elvira-Rendueles B., Gutiérrez-Bustillo M. \& Muñoz-Rodríguez A-F. 2012. Cladosporium airborne spore incidence in the environmental quality of the Iberian Peninsula. Grana, 51: 293-304.

Almaguer M., Sánchez K. \& Rojas T. 2014. El género Cladosporium en la atmósfera del Occidente de Cuba: pasado, presente y futuro. Revista Cubana de Ciencias Biológicas. 3(3), 8-17.

Andersen A. 1958. New sampler for the collection, sizing and enumeration of viable airborne particles. Journal of Bacteriology, 76(5), 471-484.

Barnett H. \& Hunter B. 1998. Illustrated Genera of Imperfect Fungi. 4th ed. APS Press, St. Paul, Minnesota.

Burch M. \& Levetin E. 2002. Effects of meteorological conditions on spore plumes. Int J Biometeorol. 46:107117. 
Calderón C., Lacey J., McCartney A. \& Rosas I. 1997. Influence of urban climate upon distribution of airborne Deuteromycetes spore concentrations in Mexico City. Int J Biometeorol. 40: 71-80.

Erkara I. P., Asan A., Yilmaz V., Pehlivan S. \& Okten S. S. 2008. Airborne Alternaria and Cladosporium species and relationship with meteorological conditions in Eskisehir City, Turkey. Environmental Monitoring and Assessment, 144(1-3), 31-41.

Grinn-Gofroń A. \& Rapiejko P. 2009. Occurrence of Cladosporium spp. and Alternaria spp. spores in Western, Northern and Central-Eastern Poland in 2004 2006 and relation to some meteorological factors. Atmos Res. 93:747-758.

Grinn-Gofroń A. \& Strzelczak A. 2012. Changes in concentration of Alternaria and Cladosporium spores during summer storms. International Journal of Biometeorology, 57(5), 759-768.

Grinn-Gofroń A. \& Bosiacka B. 2015. Effects of meteorological factors on the composition of selected fungal spores in the air. Aerobiologia, 31(1), 63-72.

Hasnain S.M. 1993. Influence of meteorological factors on the air spora. Grana. 32:184-188.

Hjelmroos M. 1993. Relationship between airborne fungal spore presence and weather variables. Grana. 32:40-47

Ianovici N., Filimon N. \& Sinitean, A. 2011. A Comparative Aeromycological Study of the Incidence of Allergenic Spores in Outdoor Environment, 18(1), 88-98.

Jones A. \& Harrison M. 2004. The effects of meteorological factors on atmospheric bioaerosol concentrations-a review. Sci Total Environ. 326:151-80.

Kasprzyk I. 2008. Aeromycology - Main research fields of interest during the last 25 years. Ann Agric Environ Med. 15:1-7.

Katial R.K., Zhang Y, Jones R.H. \& Dyer P.D. 1997. Atmospheric mold spore counts in relation to meteorological parameters. Int J Biometeorol. 41:17-22.

Kilic M., Ufuk Altintas D., Yilmaz M., Güneşer Kendirli S., Bingöl Karakoc G., Taskin E., Ceter T., Pinar N. M. 2010. The effects of meteorological factors and Alternaria spore concentrations on children sensitized to Alternaria. Allergol Immunopathol. 38(3): 122-28.

Kumari S., Gond D. K., Samuel C. O. \& Abassi P. 2011. A comparative study of aeromycospora in different localities of Gorakhpur, U. P. Indian J. Sci. Res., 2(4), $51-$ 55.

Leach C. M. 1971. A practical guide to the effects of visible and ultraviolet light on fungi. pp. 609- 664. In Booth C. (ed.) Methods in Microbiology. Vol 4. Academic Press, New York, 795p.

Lin W-H. \& Li C-S. 2000. Associations of fungal aerosols, air pollutants and meteorological factors. Aerosol Science and Technology, 32(4), 359-368.

Luo Y., Li J., Zhang X. \& Gao W. 2016. Characterization of potential pathogenic Cladosporium exposure risks from heating, ventilation and air conditioning (HVAC) in two cities, China. Medical Mycology. 2(3), 1-8.
Lyon F., Kramer C. \& Eversmeyer M. 1984. Variation of airspora in the atmosphere due to weather conditions. Grana, 23(3), 177-181.

McCallan S. \& Chan S. 1944. Inducing sporulation of Alternaria solani in culture. Boyce Thompson Inst. 13:323-336.

Peternel R., Čulig J. \& Hrga I. 2004. Atmospheric concentrations of Cladosporium spp. and Alternaria spp. spores in Zagreb (Croatia) and effects of some meteorological factors. Ann Agric Environ Med. 11:3037.

Ramsey G. \& Bailey A. 1930. Effects of ultra-violet radiation upon sporulation in Macrosporium and Fusarium. Botan. Gaz. 89:113-136.

Ríos-Yuil J., Arenas R., Fernández R., Calderón-Ezquerro M. \& Rodríguez-Badillo R. 2012. Aeromycological study at the intensive care unit of the "Dr. Manuel Gea Gonzalez" General Hospital. Brazilian Journal of Infectious Diseases, 16(5), 432-435.

Rodríguez-Rajo F., Iglesias I. \& Jato V. 2005. Variation assessment of airborne Alternaria and Cladosporium spores at different bioclimatical conditions. Mycol Res 109 (4): 497-507.

Rodríguez-Romero J., Hedtke M., Kastner C., Müller S. \& Fischer R. 2010. Fungi, hidden in soil or up in the air: Light makes a difference. Annu Rev Microbiol. 64:585610.

Sabariego S., Díaz C. \& Alba F. 2004. Estudio aerobiológico de los conidios de Alternaria y Cladosporium en la atmósfera de la ciudad de Almería (SE de España). Rev Iberoam Micol 21:121-127.

Sakiyan N. \& Inceoglu O. 2003. Atmospheric concentrations of Cladosporium Link and Alternaria Nées spores in Ankara and the effects of meteorological factors. Turk $\mathbf{J}$ Bot. 27, 77-81.

Sánchez K. \& Almaguer M. 2014. Aeromicología y salud humana. Revista Cubana de Medicina Tropical. 66(3), 322-337.

Sen B. \& Asan A. 2009. Fungal flora in indoor and outdoor air of different residential houses in Tekirdag City (Turkey): Seasonal distribution and relationship with climatic factors. Environ Monit Assess. 151:209-219.

Stępalska D. \& Wołek J. 2005. Variation in fungal spore concentrations of selected taxa associated to weather conditions in Cracow, Poland, in 1997. Aerobiologia. 21:43-52.

Tuite J. 1969. Plant pathological methods, fungi and bacteria. pp.141 - 175. Burgess Publ., Minneapolis, Minn., 239p.

Viegas C., Alves C., Carolino E., Rosado L. \& Silva Santos C. 2010. Prevalence of fungi in indoor air with reference to gymnasiums with swimming pools. Indoor Built Environ. 19(5):555-61.

Zoppas B., Valencia-Barrera R. M. \& Fernández-Gonzáles D. 2011. Distribuição de esporos de Cladosporium spp no ar atmosférico de Caxias do Sul, RS, Brasil, durante dois anos de estudo. Rev. Bras. Alerg. Imunopatol., 55-58.

\footnotetext{
${ }^{1}$ Universidad Agraria La Molina, Facultad de Ciencias, Departamento de Biología. roberto@lamolina.edu.pe.

${ }^{2}$ Universidad Agraria La Molina, Facultad de Ciencias, Departamento de Biología. vmeza@ lamolina.edu.pe.
} 